\title{
Comportamiento de morteros con áridos locales de la Zona 3 de Ecuador, durante la impresión 3D.
}

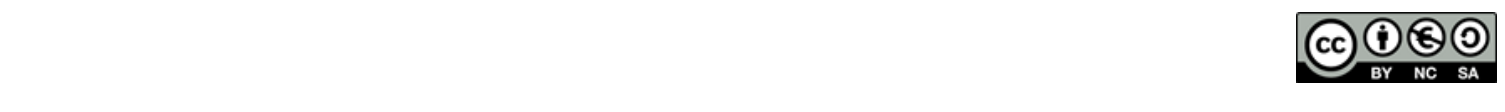

Behavior of mortars with local aggregates from Zone 3 of Ecuador, during $3 D$ printing.

\author{
Marco Javier Palacios Carvajal. ${ }^{1}$, Sandra Gissela Ortega Huilcapi. ${ }^{2}$, Dolores Amada \\ Gualli Bonilla. ${ }^{3} \&$ Mery Rossana Manzano Cepeda. ${ }^{4}$
}

\begin{abstract}
.
DOI: https://doi.org/10.33262/concienciadigital.v4i1.2.1590

Introduction. The 3D prints with cement and sand mortars were made with a fabrication printer combining with a standard type of printer the same one that was modified with an extruder to eject the mortar in a way that executes desired elements. Objective. To analyze cement mortars with sand to see their behavior during the 3D printing process, with aggregates obtained from mines in zone 3 of Ecuador. Methodology. The physical, chemical and mechanical characteristics of the materials used were previously analyzed at the National University of Chimborazo, whose results are of great importance for the analysis of the experimental tests elaborated in a prototype printer that allows this process. Results. It is evidenced that the two mortars described are printable. In addition, it is determined that for the mortar $\mathrm{CH} 1$ the material can be inside the injector up to 11 minutes and for TN1 only 7 minutes, times that can be without hardening, otherwise the printer nozzle is clogged. Conclusions. The $\mathrm{CH} 1$ mortar during printing forms more precise elements but its setting is slow and has greater deformations in each row, the TN1 elements are less precise but the setting of the mortar is faster than $\mathrm{CH} 1$ and generates less deformations.
\end{abstract}

\footnotetext{
1 Universidad Nacional de Chimborazo, Docente de la Facultad de Ingeniería. Riobamba, Ecuador. jpalacios@unach.edu.ec, https://orcid.org/0000-0002-6059-6171

${ }^{2}$ Universidad Politécnica de Madrid, Estudiante de Posgrado de la Escuela Técnica Superior de Edificación. Madrid, España. sandragissela.ortega@alumnos.upm.es, https://sandbox.orcid.org/0000-0002-4577-6983

3 Universidad Nacional de Chimborazo, Docente de la Facultad de Ingeniería. Riobamba, Ecuador. dgualli@unach.edu.ec, https://orcid.org/0000-0002-3870-7461

${ }^{4}$ Universidad Nacional de Chimborazo, Docente de la Facultad de Ingeniería. Riobamba, Ecuador. mmanzano@unach.edu.ec, https://orcid.org/0000-0001-9262-9671
} 
Keywords: 3D printing, mortars, aggregates, zone 3 of Ecuador, Universidad Nacional de Chimborazo, behavior.

\section{Resumen}

Introducción. Las impresiones 3D con morteros de cemento y arena se realizaron con una impresora de fabricación combinando con un tipo de impresora estándar la misma que fue modificada con un extrusor para que expulse el mortero de manera que ejecute elementos deseados. Objetivo. Analizar morteros de cemento con arena para ver su comportamiento durante el proceso de impresión 3D, con áridos obtenidos en minas de la zona 3 del Ecuador. Metodología. Las características físicas, químicas y mecánicas, de los materiales usados fueron analizados con anterioridad en la Universidad Nacional de Chimborazo, cuyos resultados son de gran importancia para el análisis de los ensayos experimentales elaborados en un prototipo de impresora que permite este proceso. Resultados. Se evidencia que los dos morteros descritos son imprimibles. Además, se determina que para el mortero $\mathrm{CH} 1$ el material puede llegar a estar dentro del inyector hasta 11 minutos y para TN1 únicamente 7 minutos, tiempos que pueden estar sin que estos se endurezcan caso contrario se tapona la boquilla de la impresora. Conclusiones. El mortero CH1 durante la impresión forma elementos más precisos pero su fraguado es lento y tiene mayores deformaciones en cada hilada, los elementos de TN1 son menos precisos pero el fraguado del mortero es más rápido que $\mathrm{CH} 1$ y genera menos deformaciones.

Palabras Claves: Impresión 3D, morteros, áridos, zona 3 del Ecuador, Universidad Nacional de Chimborazo, comportamiento.

\section{Introducción}

La impresión de mortero en 3D es un método de construcción que tiene la capacidad de fabricar un prediseñado de construcción en hiladas 2D una encima de la otra, cuya repetición completa un Modelo 3D (Malaed, Z., Hachem, H., et al., 2015). En comparación con métodos tradicionales, las tecnologías 3D permiten una automatización completa del proceso, reducen la cantidad de residuos, mejoran la sostenibilidad de la construcción y la expresividad arquitectónica de las estructuras, así como proporcionan solidez de las estructuras gracias a la aplicación continúa hilada por hilada (Demyanenko, O., Koponitsa N., Sorokina E., 2018).

A nivel mundial existen varias investigaciones sobre la impresión 3D en el sector de la construcción civil. Marchment, Sanjayan y Xia (2019), indican que el comportamiento del material utilizado en la técnica de impresión 3D corresponderá a las condiciones externas e internas en las que se genere la impresión, además, que cada material debe tener compatibilidad con la impresora a utilizar. Hager y Putabowicz (2016) aseguran que el desarrollo de un material apropiado para esta tecnología es el reto más grande en construir los proyectos que implican técnica 3D. 
Una vez obtenido un mortero considerado imprimible por sus características físicas y químicas, es necesario determinar dentro de un equipo que realice impresión 3D el comportamiento de sus propiedades para determinar si son materiales imprimibles.

Existen varias propiedades que debe cumplir un material imprimible durante y después de su uso en impresión, las consideradas en esta investigación son:

Capacidad de bombeo. - La dosificación del mortero debe generar una mezcla homogénea capaz de atravesar el sistema de suministro y de inyección, evitando taponamientos o corte de material.

Estabilidad de los morteros. - Se requiere que la mezcla se encuentre en un nivel que permita una estabilidad y forma adecuada de la hilada impresa (Reyes, 2018). Después de elaborada la mezcla es necesario buscar un tiempo de reposo antes de su uso en la impresión característica que ayudará a alcanzar esta condición.

Tiempo trabajable del mortero en el inyector. - Es el periodo de tiempo que el mortero permite una adecuada capacidad de bombeo y características óptimas para su impresión. Acabado superficial de las piezas. - Es la estratificación generada por la acumulación de hiladas que genera un defecto que puede apreciarse visualmente en la superficie de la pieza (Losso, 2017).

Constructibilidad. - Es la capacidad que tiene el mortero para ser construido en número de hiladas sin una deformación apreciable, se relaciona directamente con su resistencia para soportar el peso propio, peso de las hiladas superiores y presión de la inyección del mortero.

Análisis Dimensional. - Se ha utilizado para analizar los elementos después de ser impresos y conocer si los morteros permitieron crear un elemento con las características programadas en el equipo y su variación de medidas durante su proceso de fraguado.

\section{Metodología}

Para el desarrollo de esta investigación se construyó un prototipo de impresora 3D que ha sido elaborada utilizando una impresora 3D Anet A8 que utiliza filamentos plásticos para su impresión, se adaptó un extrusor usando una técnica de inyección mediante un tornillo sinfín. La impresora utilizada para los modelos digitales que fueron impresos de forma 3D, fueron elaborados en un archivo generado en AutoCad la misma que tenía una extensión. STL además fue necesario configurar la impresión en el sofware MatterControl. 
Figura 1 Prototipo de impresora 3D para morteros

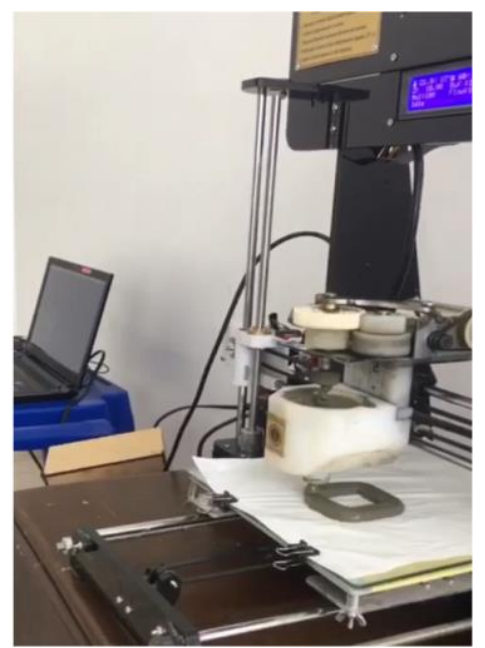

Fuente: Fotografía propia

Para esta investigación se ha seleccionado las dosificaciones con mejores características de imprimibilidad obtenidas de la indagación realizada por Mariño (2018) y Taco (2019) mostrada en la tabla 1, se utilizó agregado fino proveniente de las provincias de Chimborazo y Tungurahua respectivamente.

Tabla 1. Descripción de dosificación

\begin{tabular}{lc}
\hline Componentes & M-0-HE \\
\hline Árido Fino $(\mathrm{Kg})$ & 62.25 \\
Cemento $(\mathrm{Kg})$ & 50.00 \\
Cal $(\mathrm{Kg})$ & 0.00 \\
Agua $(\mathrm{Kg})$ & 20.75 \\
Plastificante $(\mathrm{Kg})$ & 1.00 \\
\hline
\end{tabular}

Fuente: Adaptado de Mariño (2018) y Taco (2019)

Los factores externos del lugar donde se realizaron las impresiones fueron medidas mediante un equipo armado por un Arduino con un sensor que establece la temperatura y la humedad ambiente, se tomó el registro cada segundo durante el proceso de impresión de los morteros.

Para una mejor interpretación de los resultados se utilizó las nominaciones mostradas en la tabla 2, diferenciando la localidad de cada uno de éstos.

Tabla 2. Nominación de Morteros

\begin{tabular}{cc}
\hline Nominación & Localización de la Arena \\
\hline CH1 & Provincia de Chimborazo \\
\hline TN1 & Provincia de Tungurahua
\end{tabular}

Elaborado por: Grupo de investigación

Se utilizó para los morteros cemento portland tipo HE, el árido (arena de río) de las dos provincias, para la elaboración se tomó en estado SSS (Saturado superficialmente seco) 
como lo recomienda Taco (2019), en su estudio. El estado SSS fue determinado en laboratorio según la norma INEN 856. Áridos (Determinación de la densidad relativa (gravedad específica) y absorción del árido fino).

Para conocer la capacidad de ser bombeado de los morteros se ha realizado las pruebas de impresión inmediatamente elaborado el mortero, si permite su flujo continuo se considera la dosificación como aceptable, caso contrario se procede a realizar una corrección en su dosificación, considerando estudios como el Montoya, Cadavid y Gómez (2009), además se tomó las sugerencias técnicas del plastificante utilizado. Para la corrección se permitió la variación de la cantidad de aditivo en un rango de $0.2 \%$ al $2.5 \%$ del peso del cemento con la finalidad de no alterar las características determinadas en los estudios posteriores.

La estabilidad de los morteros fueron evaluados mediante la impresión de material después de 1 minuto de elaborada la muestra, si se mantiene la configuración después de la impresión se lo considera de buena calidad la muestra caso contrario se vuelve amasar el mortero y se repite el proceso de impresión pero en este caso se espera un tiempo mayor al anterior hasta que la impresión se mantenga firme y evite el escurrimiento, al tiempo de espera se ha denominado tiempo de reposo necesario para que el mortero obtenga la condición de estabilidad.

Mediante la impresión de las hiladas de los morteros CH1 y TN1 se obtuvo la cantidad de tiempo que los morteros pueden encontrarse dentro del inyector sin que estos endurezcan.

Se estableció dos figuras cuyos modelos son mostrados en la figura 2, además se encuentra sus dimensiones y el número de hiladas para estas pruebas.

Figura 2. Modelo de los elementos propuestos
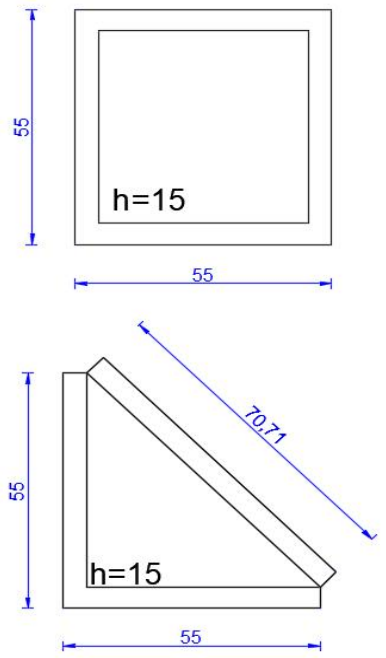

Las medidas están en mm.
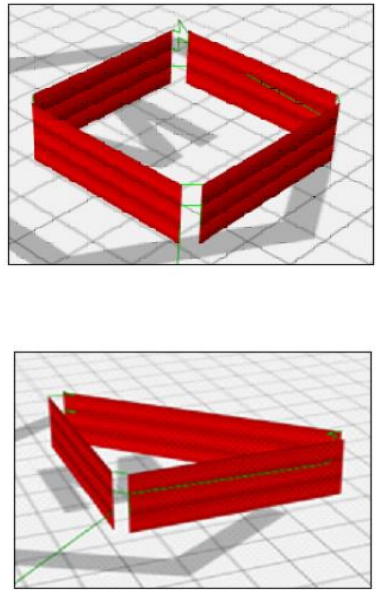

Fuente: Elaboración propia

La finalidad de esta investigación fue evaluar los morteros y más no el equipo, por lo cual los parámetros de la tabla 3 fueron fijos en todos los ensayos. 
Tabla 3. Parámetros de los ensayos

\begin{tabular}{cc} 
Velocidad de impresión: & $0.25 \mathrm{~mm} / \mathrm{seg}$ \\
Ancho y alto de la hilada: & $5 \mathrm{~mm}$ \\
Temperatura de la cama de impresión: & $60^{\circ} \mathrm{C}$ \\
\hline Elaborado por: Grupo de investigación
\end{tabular}

El acabado superficial de los elementos se analizó con la impresión de las piezas mostradas en la figura 2 conformadas con tres hiladas siguiendo una trayectoria fija, se evaluó de forma visual también se valoró la velocidad de flujo.

Para analizar la constructibidad se utilizó la norma INEN 158. Método de Vicat, la misma que indica que se controle el tiempo inicial de fraguado y su tiempo final de fraguado, de esta manera se determinó la resistencia de la pasta de cada hilada. Además, en términos de espesor se determinó la deformación de cada hilada, para esto se imprime la primera hilada y se registrar el espesor a continuación se imprime la segunda hilada y se registra nuevamente el espesor de la primera hilada y el espesor de la segunda hilada, por último, se realizó la impresión de la tercera hilada posterior a esto se tomó los espesores de la primera, segunda y tercera hiladas.

El análisis dimensional ha sido evaluado como lo recomienda Reyes (2018) en sus ensayos experimentales correspondientes a la calidad de impresión y estabilidad dimensional. Se realizó un análisis estadístico con los datos obtenidos de las longitudes de cada lado de las figuras establecidas, estas medidas fueron tomadas mediante la utilización de un calibrador. Los datos indicados fueron tomados de las hiladas el momento de su impresión y también se tomaron lecturas posteriores al fraguado del mortero.

\section{Resultados}

Según lo descrito en los resultados de los ensayos experimentales realizados en cada mortero de cada provincia se determina lo siguiente:

Condiciones externas de los ensayos. - Los resultados presentados en la tabla 4 son una estadística de la temperatura y la humedad ambiente registradas durante impresiones generadas con $\mathrm{CH} 1$ y TN1.

Tabla 4. Estadística del ensayo de Temperatura y Humedad Ambiente

\begin{tabular}{ccc}
\hline & Temperatura & Humedad \\
\hline Media & 21.604 & 50.129 \\
\hline Desviación Estándar & 0.0824 & 0.0935 \\
\hline
\end{tabular}


Elaborado por: Grupo de investigación

Figura 3. Variación de temperatura ambiente durante la impresión

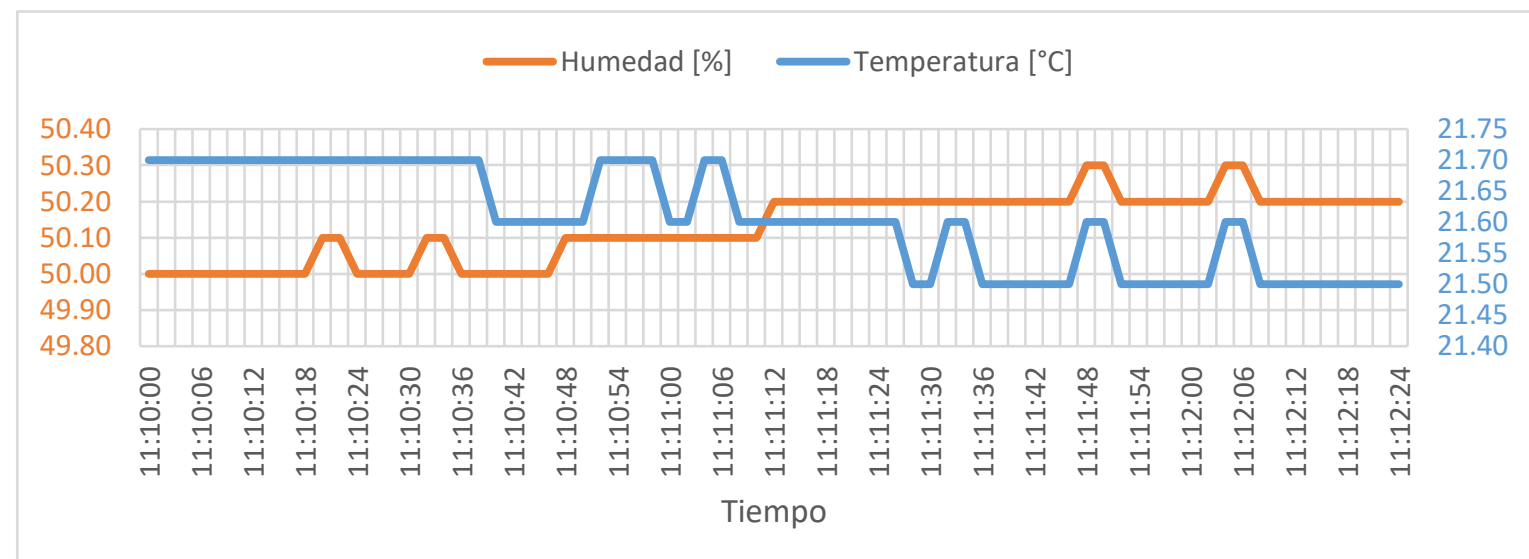

Fuente: Grupo de Investigación

En la figura 3 se muestra la variación de temperatura el momento del proceso de impresión instantánea y que además se debe tomar en cuenta que la base donde esta se imprimía se encontraba a una temperatura de $60^{\circ} \mathrm{C}$. Durante el proceso de la impresión se experimentó que mientras se genera la impresión la temperatura del ambiente tiende a disminuir mientras que la humedad ambiente aumenta, éstas son características adecuadas para utilizar el material ya que Reyes (2018) asegura que a menor temperatura provoca una mayor trabajabilidad del material, mientras que Marchment, Sanjayan y Xia (2019) demuestran que la humedad superficial aumenta la fuerza de la adhesión de las capas.

Capacidad de ser bombeado. - En la tabla 5 se muestra las dosificaciones corregidas a los morteros analizados, además fueron comprobados su compatibilidad entre estos para ser utilizados en el equipo de impresora 3D, respecto a su capacidad de ser bombeado con un flujo continuo.

Tabla 5. Dosificación corregida en el plastificante para $50 \mathrm{Kg}$ de cemento

\begin{tabular}{ccccc}
\hline Mortero & $\begin{array}{c}\text { Arena } \\
(\mathbf{K g})\end{array}$ & $\begin{array}{c}\text { Cemento } \\
(\mathbf{K g})\end{array}$ & $\begin{array}{c}\text { Agua } \\
(\mathbf{K g})\end{array}$ & $\begin{array}{c}\text { Aditivo } \\
\text { Plastificante } \\
(\mathbf{K g})\end{array}$ \\
\hline CH1 & 62.25 & 50 & 20.75 & 1 \\
\hline TN1 & 62.25 & 50 & 20.75 & 2 \\
\hline
\end{tabular}

Elaborado por: Grupo de investigación

Como se puede observar en la tabla 5 el mortero TN1, necesitó más cantidad de plastificante debido a que durante la impresión causó un bloqueo y disminución de la velocidad del flujo, pero luego del análisis se encontró que el arena de río de la provincia de Tungurahua notándose que se requiere mayor cantidad de aditivo porque su 
particulado es de mayor granulometría, con esta modificación se puedo obtener mejores propiedades en la impresión 3D.

Estabilidad de los morteros. - La figura 4 y 5 que se detallan a continuación indican la extrusión de CH1 y TN1, en la cual indican la estabilidad de los morteros respecto a un tiempo de espera que se encontraba el mortero dentro del inyector para que luego de la impresión poder observar si poseen una adecuada estabilidad cada mortero.

Figura 4. Mortero $\mathrm{CH} 1$ (Estabilidad vs Tiempo)

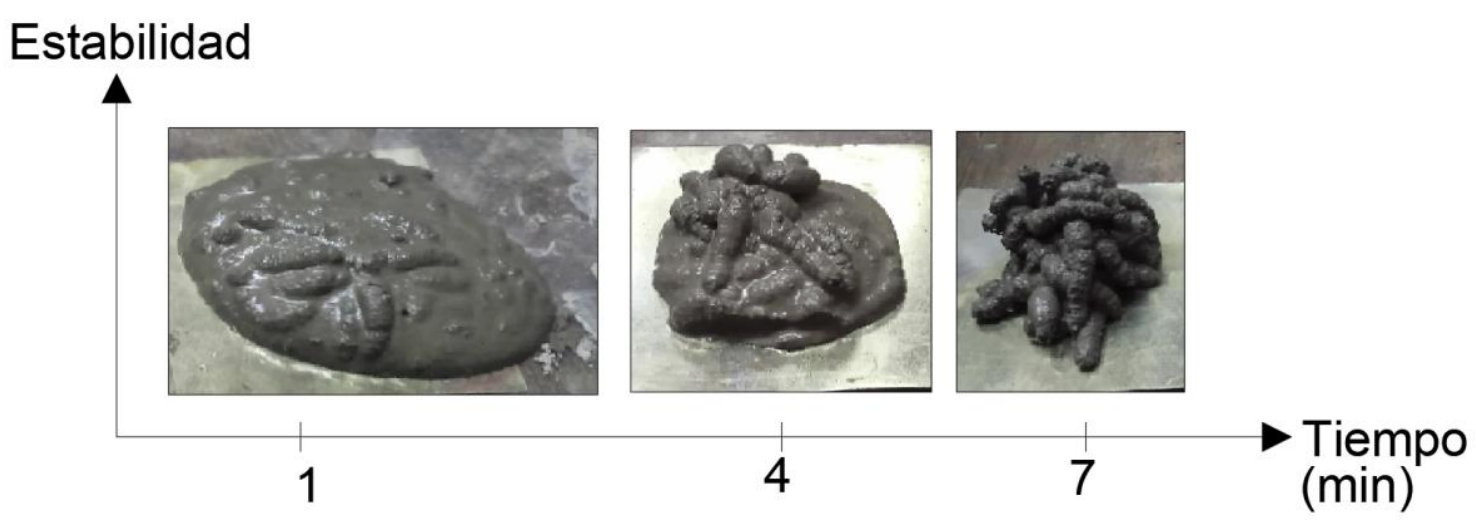

Fuente: Grupo de Investigación

Figura 5. Mortero TN1 (Estabilidad vs Tiempo)

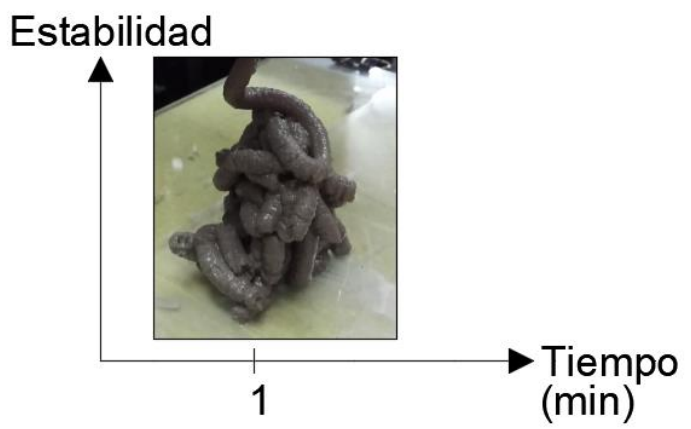

Fuente: Grupo de Investigación

En la figura 4, si observamos la línea de tiempo realizada para el mortero $\mathrm{CH} 1$ evidencia que requiere un tiempo de reposo dentro del inyector de 7 minutos antes de ser utilizada en la impresión para evitar el escurrimiento, mientras que en la figura 5 el comportamiento del mortero TN1 tiene una correcta estabilidad al ser extruido en el primer minuto de elaborada la muestra por lo que se confirma que cuando se utiliza una cantidad mayor de aditivo plastificante la estabilidad aumenta y disminuye el escurrimiento.

Tiempo que los morteros pueden encontrarse dentro del inyector. - Los resultados se muestran en la tabla 6 , corresponden a una estadística de varias experiencias realizadas durante varios ensayos colocando morteros dentro del inyector. 
Tabla 6. Tiempo que los morteros pueden encontrarse dentro del inyector

\begin{tabular}{cc}
\hline Mortero & Tiempo (min) \\
CH1 & 11 \\
TN1 & 7 \\
\hline
\end{tabular}

Elaborado por: Grupo de investigación

La tabla 6 indica que $\mathrm{CH} 1$ el mortero puede estar dentro del inyector como máximo 11 minutos sin que este se endurezca mientras que TN1 el tiempo máximo que puede estar dentro del inyector sin que este se endurezca es de 7 minutos, por lo tanto, los dos morteros son óptimos ya que cumplen con el objetivo mencionado por Reyes (2018) el de poseer suficiente tiempo con la trabajabilidad necesaria para elaborar el producto final que se desea imprimir.

Calidad de impresión. - El resultado de la calidad de impresión de CH1 y TN1 se muestran en la figura 6 y figura 7 respectivamente.

Figura 6. Calidad de impresión mortero $\mathrm{CH} 1$

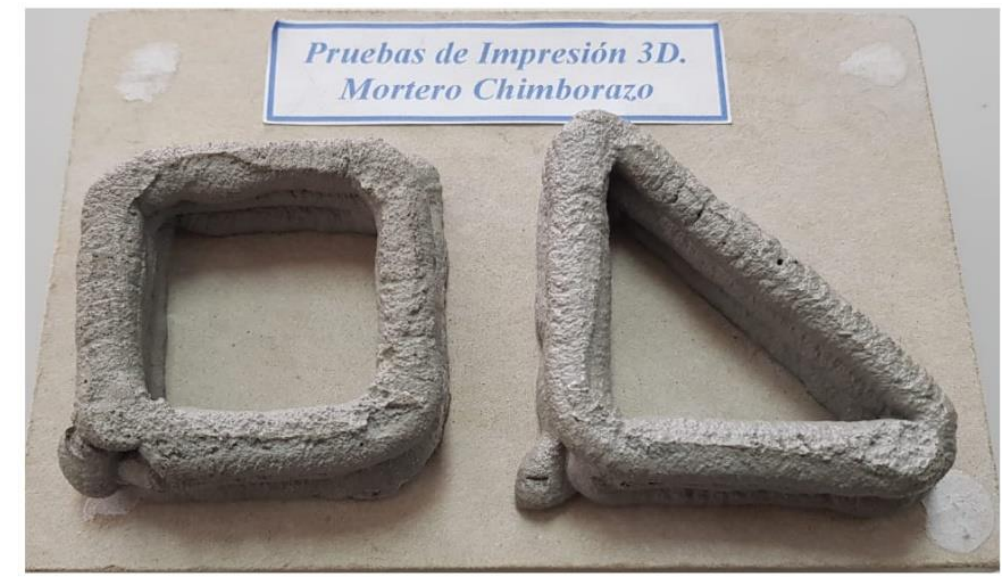

Fuente: Grupo de Investigación

Figura 7. Calidad de impresión mortero TN1

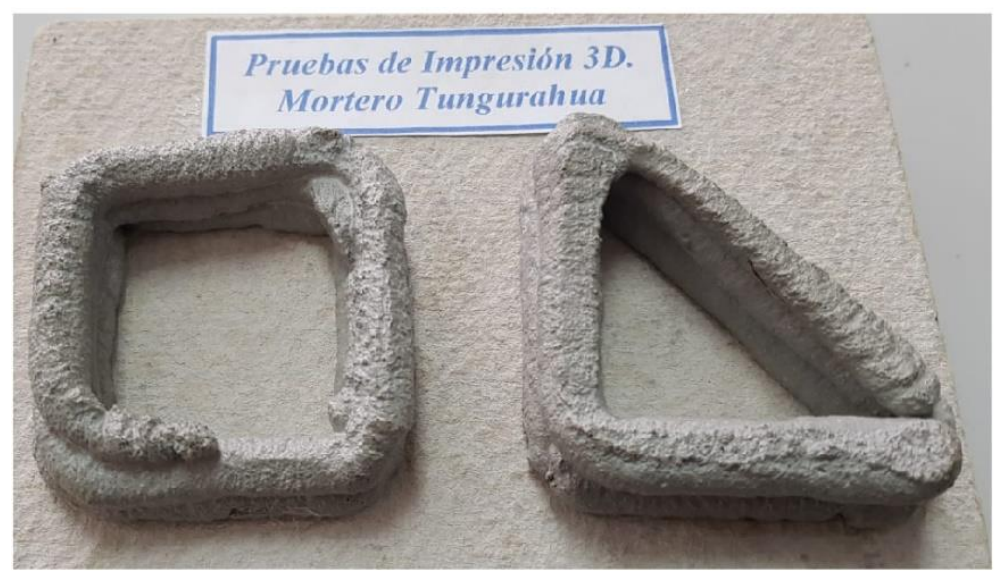

Fuente: Grupo de Investigación 
Los morteros $\mathrm{CH} 1$ y TN1 como se muestra en la figura 6 y 7 cumplen correctamente con la fluidez necesaria, no generan bloqueo y obstrucción, pero los dos morteros crearon problemas el momento de entregar el flujo, sin embargo, esto fue controlada mediante el aumento de potencia al inyector del equipo. Se observó que tanto $\mathrm{CH} 1$ como TN1 luego de la impresión generan lo denominado por Buswell, et al. (2018), rotura y división del material que son fisuras de tipo microscópicas que comparando entre las dos muestras la que presenta menor cantidad es $\mathrm{CH} 1$, no obstante, se acepta ésta condición debido a lo mencionado por Russel (2018) quien indica que éste comportamiento es una característica de la extrusión mediante una boquilla con un tornillo mezclador similar al equipo utilizado en nuestra investigación.

Constructibilidad - Los resultados de los ensayos de Vicat se encuentran representados en la tabla 7 con los valores de los tiempos medios, obtenidos del proceso de fraguado posterior a la impresión.

Tabla 7. Tiempo inicial y final de fraguado posterior a la impresión

\begin{tabular}{ccc}
\hline Mortero & $\begin{array}{c}\text { Tiempo Inicial de } \\
\text { fraguado }(\text { min) }\end{array}$ & $\begin{array}{c}\text { Tiempo Final de } \\
\text { fraguado (min) }\end{array}$ \\
\hline CH1 & 15 & 35 \\
TN1 & 14 & 33 \\
\hline & Elaborado por: Grupo de investigación
\end{tabular}

En la tabla 7 se muestra los valores de los tiempos medios del fraguado inicial y final considerando que la placa base donde se realizó la impresión se encontraba a una temperatura de $60^{\circ} \mathrm{C}$, de los resultados determinados en la impresión si comparamos con los resultados encontrados en laboratorio por Mariño (2018) y Taco (2019) estos son menores, por lo que se concluye que el aumento de temperatura en la cama de impresión reduce el tiempo de fraguado y ayuda a un proceso de construcción acelerado, éste es un parámetro que debe ser analizado a fondo conjuntamente con la capacidad de la adhesión de las hiladas obtenido a partir del ensayo de tracción directa uniaxial como lo menciona Marchment, et al. (2019), ya que mientras mayor sea el tiempo de colocación entre hiladas la adhesión de las mismas será menor.

Para el análisis de las deformaciones de hiladas se muestra la tabla 8 correspondiente al análisis estadístico de las deformaciones obtenidas en términos de espesor. La deformación de la hilada 1 se genera al soportar una sola hilada superior, mientras tanto la deformación 2 es generada por la colocación de dos hiladas superiores sobre la primera. Para la lectura de la hilada 2 se tomó una sola deformación la misma que es cuando actúa una hilada sobre ésta. 
Tabla 8. Análisis estadístico para deformaciones de hiladas

\begin{tabular}{|c|c|c|c|c|}
\hline Mortero & Hilada & Medida & $\begin{array}{l}\text { Deformación } 1 \\
(\mathrm{~mm})\end{array}$ & $\begin{array}{l}\text { Deformación } 2 \\
(\mathrm{~mm})\end{array}$ \\
\hline \multirow{4}{*}{$\mathrm{CH} 1$} & \multirow{2}{*}{1} & Media & 0.714 & 0.5333 \\
\hline & & Desv. Est. & 0.510 & 0.4247 \\
\hline & \multirow{2}{*}{2} & Media & 0.8619 & \\
\hline & & Desv. Est. & 0.3612 & \\
\hline \multirow{4}{*}{ TN1 } & \multirow{2}{*}{1} & Media & 0.581 & 0.4571 \\
\hline & & Desv. Est. & 0.304 & 0.4020 \\
\hline & \multirow{2}{*}{2} & Media & 0.6714 & \\
\hline & & Desv. Est. & 0.1554 & \\
\hline
\end{tabular}

Tanto para CH1 y TN1 en las experimentaciones se notó que la hilada dos resiste mayor deformación en relación a la hilada 1 , determinando este comportamiento al efecto de encontrarse la hilada directamente en contacto con la cama de impresión que estaba a una temperatura de $60^{\circ} \mathrm{C}$ por lo que acelera su proceso de endurecimiento. Este parámetro de temperatura en la cama es bueno porque ayuda a fraguar más rápido al mortero de la primera hilada y de esta manera soportar el peso de las hiladas superiores con menores deformaciones. Sin embargo, se debe tomar en cuenta que éste es un parámetro es significativo el momento que se realice una impresión a escala real.

La tabla 9 muestra la estadística de la deformación total de la primera hilada en varios ensayos de impresiones de elementos con 3 hiladas.

Tabla 9. Estadística de la deformación total de la primera hilada

\begin{tabular}{ccc}
\hline Mortero & Medida & Deformación Total capa $\mathbf{1}(\mathbf{m m})$ \\
\hline \multirow{2}{*}{ CH1 } & Media & 1.171 \\
& Desv. Est. & 0.554 \\
\hline \multirow{2}{*}{ TN1 } & Media & 1.114 \\
& Desv. Est. & 0.416
\end{tabular}

Elaborado por: Grupo de investigación

De éste análisis en el que cada hilada tiene un tiempo aproximado de reposo de 1 minuto sobre la cama de impresión para estabilizarse y soportar el peso de una nueva hilada, como se indica en la tabla 9, el mortero TN1 tiene una menor deformación que CH1. Se debe tener en cuenta que este análisis puede tener variación a medida que se aumente las hiladas o las condiciones varían.

Análisis Dimensional. - La tabla 10 corresponde a la parte estadística representada de 54 muestras de las dimensiones de las figuras impresas. 
Tabla 10. Análisis Dimensional de los morteros luego del proceso de impresión en 3D

\begin{tabular}{cccc}
\hline & Medida & CH1 & TN1 \\
\hline $\begin{array}{c}\text { Estabilidad } \\
\text { Dimensional }(\mathbf{m m}) \\
\begin{array}{c}\text { Conformidad } \\
\text { Dimensional }(\mathbf{m m})\end{array}\end{array}$ & Media & 0.775 & -0.208 \\
\hline
\end{tabular}

Elaborado por: Grupo de investigación

La estabilidad dimensional se encuentra representada con la diferencia de las dimensiones de los elementos recién impresos con las dimensiones de los elementos cuando han fraguado, por lo tanto la estabilidad dimensional deseada se considera 0 porque representa que las dimensiones de las hiladas durante la impresión se mantiene con la misma forma como fue depositado por el equipo, notándose que cuando los valores están de manera positivos significa que la muestra se ha contraído, mientras que los valores negativos significa que la muestra está expandida. Como muestra la tabla 10, los ensayos realizados para $\mathrm{CH} 1$ indica que los elementos impresos luego de fraguar tienden a contraerse por lo tanto los valores en la tabla son positivos lo que corresponde a una característica general de un mortero en impresión 3D según Reyes (2018), si comparamos con TN1 ocurre lo opuesto a la media de sus ensayos que establecen que sus hiladas tienden a expandirse.

La conformidad dimensional corresponde a la capacidad del mortero de pasar las medidas programadas en el software versus las medidas luego de la impresión. Los valores de $54.87 \mathrm{~mm}$ para el mortero CH1 y $54.62 \mathrm{~mm}$ para TN1 corresponden a la media de varias muestras impresas con una dimensión programada en el software de $55 \mathrm{~mm}$, por consecuencia se concluye que los morteros permiten imprimirse con dimensiones dentro de la tolerancia del 10\% aceptada y permitida según la investigación de Reyes (2018). Comparando los dos morteros, el mortero $\mathrm{CH} 1$ permite imprimir elementos en 3D con dimensiones más precisas que TN1.

\section{Discusión}

Los resultados de los ensayos experimentales antes mencionados y realizados con $\mathrm{CH} 1 \mathrm{y}$ TN1 presentaron condiciones aceptables para ser impresos, además han permitido generar los elementos previstos dentro de la metodología propuesta. No obstante, se compendia que el comportamiento de $\mathrm{CH} 1$ denominado mortero elaborado con agregado fino de la provincia de Chimborazo presentó los mejores resultados en las condiciones establecidas en esta investigación, también si consideramos el equipo propuesto en este caso la impresora 3D que fue un modelo adaptado para las condiciones de esta investigación además el comportamiento fue aceptable como lo habíamos propuesto y además comparte características citadas en varias bibliografías.

\section{Conclusiones}

- Del análisis de la dosificación corregida en el plastificante se determinó que TN1 necesitó un aumento en la cantidad de aditivo respecto a la dosificación inicial 
para lograr una adecuada capacidad de bombeo, mientras que la dosificación inicial propuesta de $\mathrm{CH} 1$ cumplió correctamente las características necesarias para un mejor comportamiento dentro del inyector de la impresora.

- Respecto a la impresión podemos citar que los morteros para su uso y dentro del inyector deben ser impresos en tiempos diferentes, caso contrario tienden a fraguarse dentro del mismo, para esto se llegó a establecer que para $\mathrm{CH} 1$ su tiempo máximo que puede estar en el interior del inyector es de 11 minutos, mientras que TN1 únicamente 7 minutos. Caso contrario si pasan de estos valores de tiempo estos se fraguan ocasionando taponamiento en la boquilla de la impresora.

- Con respecto a la conformidad dimensional podemos establecer que $\mathrm{CH} 1$ permitió formar elementos más precisos con respecto a lo programado en el software en este caso fue de $55 \mathrm{~mm}$, mientras que TN1 también permitió formar elementos que no tienen gran diferencia con respecto a $\mathrm{CH} 1$ por lo tanto los dos morteros dentro de su dimensionamiento de impresión entran en el rango del $10 \%$ de aceptación de acuerdo a varias investigaciones.

- Del análisis realizado a las dimensiones de los elementos formados posterior a la impresión se obtuvo que $\mathrm{CH} 1$ permitió formar elementos más preciosos pero su tiempo de fraguado es lento lo que provoca mayores deformaciones, mientras que TN1 también forma elementos un poco menos precisos con respecto a la anterior pero su tiempo de fraguado es más rápido generando menores deformaciones. Además, los morteros $\mathrm{CH} 1$ como TN1 se determinaron visualmente que el acabado superficial presentó buenas condiciones monolíticas.

\section{Referencias}

Buswell R., Leal W.R., Jones S.Z. (2018). 3D impresión que utiliza extrusion concreta. ElSevier.

Demyanenko, O., Koponitsa N., Sorokina E. (2018). Mortars for 3D printing. MATEC Web of Conferences.

Hager I., Golonka A., Putanowics R. (2016). 3D printing of buildings and building components as the future of sustainable construction? ScienceDirect Elsevier.

Losso, H. (2017). Dosificación de mortero cementicio para impresión 3D. Cordova: Universidad de Cordova.

Malaed, Z., Hachem, H., et al. (2015). 3D Concrete printing: Mechine and mix design. International Journal of Civil Engineering and Technology (IJCIET), 14-22.

Marchment T., Sanjayan J., Xia M. (2019). Method of enhancing interlayer bond strength in construction scale 3D printing with mortar by effective bond area amplification. ScienceDirect El sevier. 
Mariño, E. (2018). Diseño de un mortero con cemento portland tipo I y HE incluida cal hidraulica para su uso en impresoras 3D. Riobamba: Universidad Nacional de Chimborazo.

Montoya Y., Cadavid A., Gomez M. (2009). Comportamiento mecanico y de fraguado de morteros de cemento portland gris con aditivos. Revista EIA, ISSN 1794-1237, 39-49.

Reyes, H. (2018). Estudio de las caracteristicas del hormigo para su uso en una maquina de impresión. Santiago de Chile: Universidad de Chile.

Roussel, N. (2018). Cement and concrete research. Institute for Building Materials, ETH Zürich, Zürich, Switzerland.

Taco, P. (2019). Diseño de un mortero con arena de la provincia de Tungurahua, utilizando cemento tipo 1 y HE incluido Cal para uso de impresoras 3D. Riobamba: Unach.

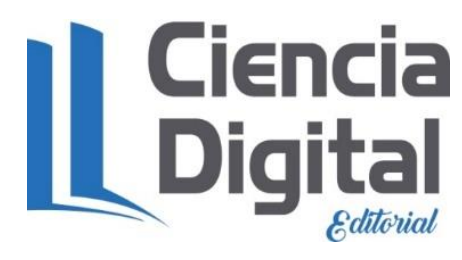


PARA CITAR EL ARTÍCULO INDEXADO.

Palacios Carvajal, M. J., Ortega Huilcapi, S. G., Gualli Bonilla, D. A., \& Manzano Cepeda, M. R. (2021). Comportamiento de morteros con áridos locales de la Zona 3 de Ecuador, durante la impresión 3D . ConcienciaDigital, 4(1.2), 220-234. https://doi.org/10.33262/concienciadigital.v4i1.2.1590

\section{\Ciencia}

El artículo que se publica es de exclusiva responsabilidad de los autores y no necesariamente reflejan el pensamiento de la Revista Conciencia Digital.

El artículo queda en propiedad de la revista y, por tanto, su publicación parcial y/o total en otro medio tiene que ser autorizado por el director de la Revista Conciencia Digital.

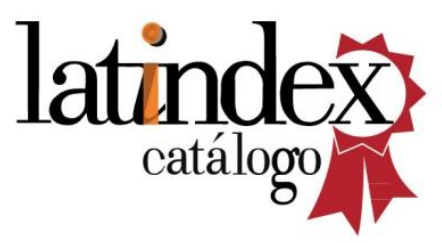

